\title{
DIFFUSION AND PERMEATION OF DEUTERIUM IN EUROFER97: EFFECT OF IRRADIATION AND OF IMPLANTED HELIUM
}

\author{
Z. YAO, C. LIU, and P. JUNG* \\ Institut für Festkörperforschung, Forschungszentrum Jülich, D-52425 Jülich, Germany
}

Received October 4, 2004

Accepted for Publication January 14, 2005

Permeability, diffusivity, and solubility of deuterium in the low-activation martensitic stainless steel EUROFER97 were derived from measurements of gas permeation in the transient and steady-state regimes at temperatures from 100 to $350^{\circ} \mathrm{C}$ and at pressures from $2 \times 10^{3}$ to $2 \times 10^{5} \mathrm{~Pa}$. The specimens were used in four conditions to investigate the effect of irradiation-induced defects: standard annealed condition, preirradiated with protons, implanted with helium, and implanted plus annealed to produce helium bubbles. In general, displacement defects as well as implanted helium tend to decrease permeation and diffusivity. Permeation and diffusion measurements were also performed under simultaneous irradiation, showing no net effect if the slight temperature increase due to irradiation is taken into account. Diffusion measurement of implanted hydrogen gave equal or slightly lower values than gas permeation, which is in qualitative agreement with results from preirradiated specimens. Trapping parameters are derived by a detailed comparison to a saturable-trap model. Results are compared to previous studies on $7 \% \mathrm{Cr} F 82 \mathrm{H}$ and $11 \% \mathrm{Cr}$ MANET-II steels, and effects of compositional variations are indicated.

KEYWORDS: permeation, diffusion, hydrogen

\section{INTRODUCTION}

Structural materials in future fusion reactors will be loaded with hydrogen isotopes by nuclear transmutation, by implantation from the plasma, and by permeation from breeding materials and coolants. ${ }^{1}$ Retention and further distribution of the hydrogen isotopes depend on permeability and diffusivity and will have implications on

*E-mail: p.jung@fz-juelich.de material stability, environmental safety, and recycling to the plasma. Problems of hydrogen on material properties, e.g., ductility, are considered more severe in ferriticmartensitic than in austenitic steels because of lower solubility and higher diffusivity of hydrogen. It is the aim of the present work to study permeability and diffusivity of hydrogen isotopes from the gas phase and from implantation in low-activation EUROFER97, with emphasis on influences of irradiation-induced displacement defects and of helium, which next to hydrogen is the most important transmutation product.

\section{EXPERIMENT}

\section{II.A. Preirradiation and He Implantation}

The reduced activation martensitic steel EUROFER97 is used in the European fusion materials program. Its major constituents in weight percent are $\mathrm{C}, 0.11 ; \mathrm{N}$, 0.03; P, 0.005; V, 0.2; Cr, 9.0; Mn, 0.48; Fe, 89.0; Ni, $0.021 ; \mathrm{Nb}, 0.0017$; Ta, 0.07; and W, 1.1. Circular specimens of 21.5-mm diameter were cut by spark erosion and polished to thicknesses from 380 to $750 \mu \mathrm{m}$. The lower thicknesses were selected because of the limited range of 28-MeV alpha particles from the Jülich Compact Cyclotron for He implantation and by the desire to reduce the time for preirradiation and implantation. Previous studies $^{2,3}$ have shown that thicknesses in this range are sufficient to approach bulk behavior.

For preirradiation, $380-\mu \mathrm{m}$-thick specimens were fixed to a copper heat sink by Wood's metal, which limited specimen temperature during irradiation and unmounting to $71^{\circ} \mathrm{C}$. Proton energy behind a $30-\mu \mathrm{m}$-thick $\mathrm{Al}$ window was $15.8 \mathrm{MeV}$, which according to the TRIMSRIM code ${ }^{4}$ results in a range of $570 \mu \mathrm{m}$ in steels, i.e., far beyond thickness. For a displacement energy $T_{d}=$ $40 \mathrm{eV}$ and a binding energy $E_{b}=2 \mathrm{eV}$, TRIM gives an average production of 16.1 defects per transmitted ion, corresponding to a displacement cross section of $0.5 \times$ $10^{-24} \mathrm{~m}^{2}$. Homogeneity of damage was achieved by 
turning the specimen after half the total dose of 0.01 displacement per atom (dpa). The irradiated area was limited by an $18-\times 18$-mm four-quadrant aperture, which sufficiently exceeded the 12 -mm-diam opening for permeation measurements. The current density was 0.03 $\mathrm{A} / \mathrm{m}^{2}$, corresponding to a displacement rate of $\approx 1.0 \times$ $10^{-7} \mathrm{dpa} / \mathrm{s}$. The beam current was measured on a shutter during beam-off periods. The beam stability during irradiation was monitored by the signals on the aperture. The measured beam current was corrected for secondary electrons by a factor of 0.7 . The beam was scanned across the specimens at $\sim 250 \mathrm{~Hz}$, spreading $>50 \%$ of the beam to the four quadrants to obtain fairly uniform distribution on the irradiated area.

Helium was implanted into $500-\mu$ m-thick specimens fixed to a heat sink, using ${ }^{3} \mathrm{He}^{++}$ions of $35.2 \mathrm{MeV}$ that have a range of $\sim 250 \mu \mathrm{m}$ in steel. A degrader wheel with 51 aluminum foils of various thicknesses was used to achieve a homogeneous distribution. When reaching half the final dose, the specimens were turned around to obtain implantation over the full thickness. By this procedure, defect production decreased from about 170 defects/ion on both surfaces to less than 100 in the center, giving an average of 130 displacement defects per implanted ion ( 0.77 at.\% He/dpa), i.e., a total of 0.065 dpa for 500 appm He. Other details conform to the case of preirradiation, with more details given in Refs. 2 and 3. Some helium-implanted specimens were annealed in vacuum for $10 \mathrm{~h}$ at $750^{\circ} \mathrm{C}$. By this treatment, helium agglomerated into bubbles with typical diameters of $5 \mathrm{~nm}$, located mainly within the grains, as observed by transmission electron microscopy.

\section{II.B. Permeation Experiment}

Permeability and diffusivity were derived from transient and stationary gas permeation experiments at temperatures from 100 to $350^{\circ} \mathrm{C}$ and upstream pressures from $10^{3}$ to $2 \times 10^{5} \mathrm{~Pa}$. The upstream gas volume was bounded on one side by a $25-\mu \mathrm{m}$-thick Hasteloy window, allowing simultaneous irradiation or implantation during permeation measurements. In this case the beam passed through an 8-mm-diam aperture, i.e., slightly smaller than the permeation area of 12-mm diameter. A cold trap filled with a molecular sieve and cooled by dry ice was attached to the gas volume. This arrangement was found optimum to effectively trap oxygen and nitrogen without affecting the hydrogen inventory. In 750- $\mu \mathrm{m}$-thick specimens, diffusion of implanted protons was measured at various implantation depths as defined by proton energy. The specimen temperature was monitored by two thermocouples located close to the specimen surface. As the thermocouples were placed outside the beam area, temperature changes during irradiation or implantation could be estimated only by heat transfer calculations. The permeation flux to the downstream volume was measured by a quadrupole mass spectrometer under continuous pumping at a rate of $\sim 2 \times 10^{-3} \mathrm{~m}^{3} / \mathrm{s}$ (Refs. 2 and 3). Apparent permeability $P^{*}$ and diffusivity $D^{*}$ are derived as described in Ref. 2.

\section{RESULTS}

\section{III.A. Permeability}

Apparent permeabilities $P^{*}$ of $\mathrm{D}_{2}$ through 500- and $750-\mu \mathrm{m}$-thick specimens of EUROFER97 in standard condition are given in Fig. 1 as a function of temperature. Included are results from $380-\mu \mathrm{m}$-thick specimens preirradiated to $0.01 \mathrm{dpa}$, from specimens implanted to 500 appm $\mathrm{He}$, and from He-implanted specimens after annealing at $750^{\circ} \mathrm{C}$ for $10 \mathrm{~h}$. Figure 1 shows that permeability is reduced by preirradiation as well as by $\mathrm{He}$ implantation, and even more by the formation of helium bubbles. Surface effects on permeation were negligible, as seen by the close agreement of the 500- and 750- $\mu \mathrm{m}$ data and more clearly by the very small pressure dependence of permeability shown in Fig. 2. Significant effects of pressure are observed only at the lowest temperatures and pressures. This is similar to the case of $11 \% \mathrm{Cr}$ MANET-II (Ref. 2), while 7\%Cr F82H-mod (Ref. 3) showed significant pressure dependence, which may be

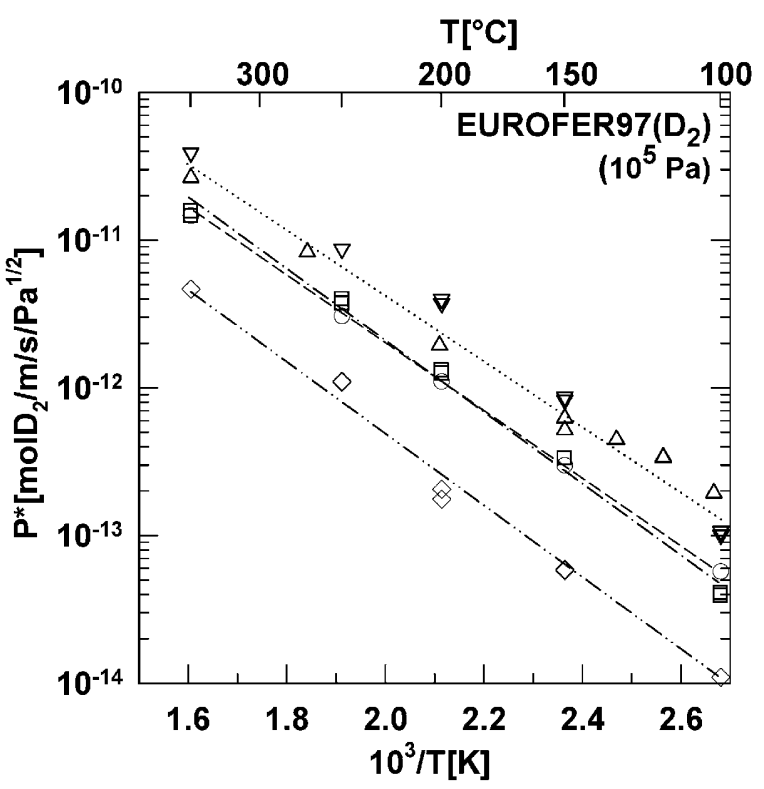

Fig. 1. Temperature dependence of apparent permeability of deuterium at $\approx 10^{5} \mathrm{~Pa}$ in $500-\mu \mathrm{m}$-thick $(\nabla, \cdots)$ and 750 - $\mu$ m-thick $(\triangle, \cdots)$ EUROFER97 of standard condition, in $380-\mu \mathrm{m}$-thick specimens preirradiated to $0.01 \mathrm{dpa}(\mathrm{O},---)$, in $500-\mu \mathrm{m}$-thick specimens preimplanted to $500 \mathrm{appm} \mathrm{He}(\square,-\cdot-)$, and in $\mathrm{He}$ implanted specimens annealed for $10 \mathrm{~h}$ at $750^{\circ} \mathrm{C}(\diamond$, -..-). Lines correspond to expressions given by Eqs. (1a) through (1d). 


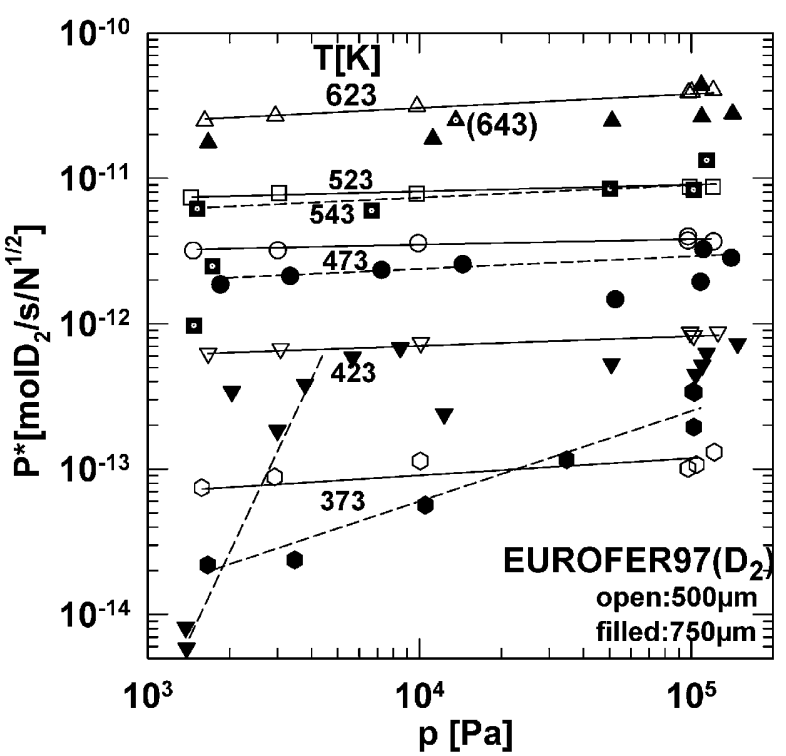

Fig. 2. Pressure dependence of apparent permeability $P^{*}$ of deuterium in EUROFER97 of standard condition with thicknesses of $500 \mu \mathrm{m}$ (open symbols) and $750 \mu \mathrm{m}$ (filled) at various temperatures. Some lines are included to guide the eye.

ascribed to surface oxides due to its lower chromium content. $^{5}$ The apparent permeabilities of $\mathrm{D}_{2}$ in EUROFER97 from 100 to $350^{\circ} \mathrm{C}$ at pressures of $\sim 10^{5} \mathrm{~Pa}$ can be roughly approximated by an Arrhenius-type dependence on temperature (straight lines in Fig. 1):

$$
\begin{gathered}
P_{(\text {std. cond. })}^{*}=1.4 \times 10^{-7} \exp (-43600 / R T), \\
P_{(0.01 \mathrm{dpa})}^{*}=7.0 \times 10^{-8} \exp (-43600 / R T), \\
P_{(500 \text { appm He })}^{*}=1.5 \times 10^{-7} \exp (-46600 / R T),
\end{gathered}
$$

and

$$
P_{(\text {He } \text { annealed })}^{*}=3.2 \times 10^{-8} \exp (-46200 / R T) .
$$

\section{III.B. Permeation Under Simultaneous Irradiation}

Permeation of $\mathrm{D}_{2}$ through a $750-\mu \mathrm{m}$-thick specimen was also measured when a beam of $20-\mathrm{MeV}$ protons with $0.01 \mathrm{~A} / \mathrm{m}^{2}$ simultaneously passed through the $\mathrm{D}_{2}$ gas on the upstream side and the specimen and when a beam of $7-\mathrm{MeV}$ protons was stopped in the specimen, respectively. No change of permeability by irradiation was observed if the slight increase in temperature due to irradiation was taken into account. Heat transfer calculations gave temperature increases of $\approx 16 \mathrm{~K}$ at $150^{\circ} \mathrm{C}$ and $\approx 5 \mathrm{~K}$ at $350^{\circ} \mathrm{C}$, respectively. A detailed analysis showed also that the apparent enhancement of permeability under irradiation reported in Refs. 2 and 3 must also be ascribed to a temperature increase due to irradiation.

\section{III.C. Diffusivity}

Diffusion coefficients $D^{*}$ of $\mathrm{D}_{2}$ in 500- and 750- $\mu \mathrm{m}$ thick specimens obtained from permeation measurements during pressure transients at pressures of $\sim 1$ bar are shown in Fig. 3 as a function of temperature. Included are the results of preirradiated and of He-implanted specimens. Similarly to permeability, only a minor unsystematic effect of specimen thickness was observed. Again, diffusivity is decreased by preirradiation and even more by helium implantation. But, diffusivity of the implanted specimens is slightly enhanced after annealing. Virgin and preirradiated material show steeper temperature dependences below $250^{\circ} \mathrm{C}$, which in Fig. 3 are crudely approximated by straight lines. This change of slope is not as clear for the He-implanted and -annealed specimens, at least in the investigated temperature range from 100 to $350^{\circ} \mathrm{C}$. The apparent asymptotic diffusivities of $\mathrm{D}_{2}$ in EUROFER97 at temperatures above $250^{\circ} \mathrm{C}$ and pressures of $\sim 10^{5} \mathrm{~Pa}$ can be described by

$$
\begin{aligned}
D_{(\text {std. cond. })}^{*} & =6.6 \times 10^{-8} \exp (-12800 / R T), \\
D_{(0.01 \mathrm{dpa})}^{*} & =6.6 \times 10^{-7} \exp (-26500 / R T), \\
D_{(500 \mathrm{appm} \mathrm{He})}^{*} & =1.5 \times 10^{-6} \exp (-39500 / R T),
\end{aligned}
$$

and

$$
D_{(\text {He annealed })}^{*}=1.2 \times 10^{-6} \exp (-35000 / R T) .
$$

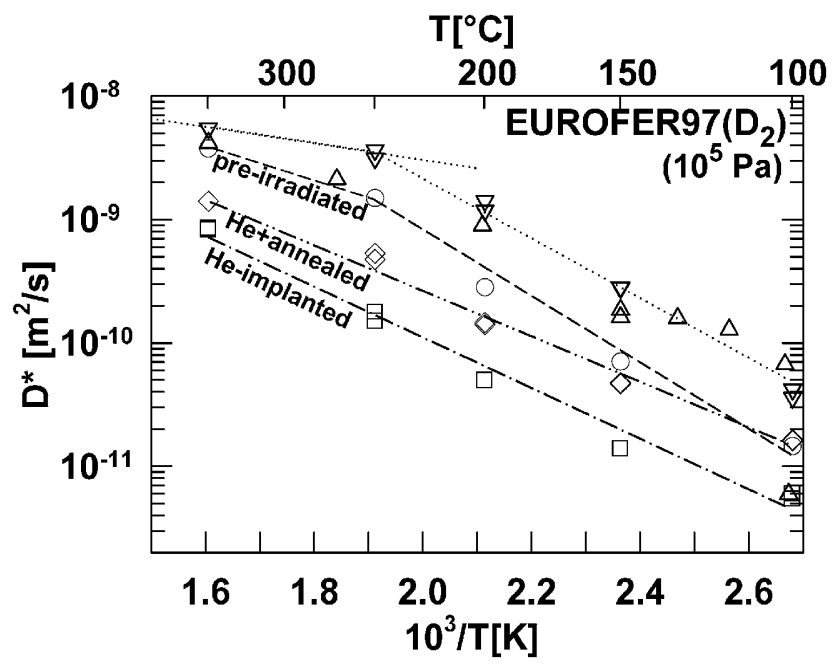

Fig. 3. Temperature dependence of apparent diffusion coefficients $D^{*}$ of deuterium at $\approx 10^{5} \mathrm{~Pa}$ in $500-\mu \mathrm{m}$-thick $(\nabla, \cdots)$ and $750-\mu$ m-thick $(\triangle, \cdots)$ EUROFER97 of standard condition, in 380- $\mu \mathrm{m}$-thick specimens preirradiated to $0.01 \mathrm{dpa}\left(\mathrm{O}_{----)}\right.$, in $500-\mu \mathrm{m}$-thick specimens preimplanted to $500 \mathrm{appm} \mathrm{He}(\square,-\cdot-)$, and in He-implanted specimens annealed for $10 \mathrm{~h}$ at $750^{\circ} \mathrm{C}$ $(\diamond,-\cdots-)$. Straight lines at the higher temperatures correspond to the expressions given by Eqs. (2a) through (2d). 


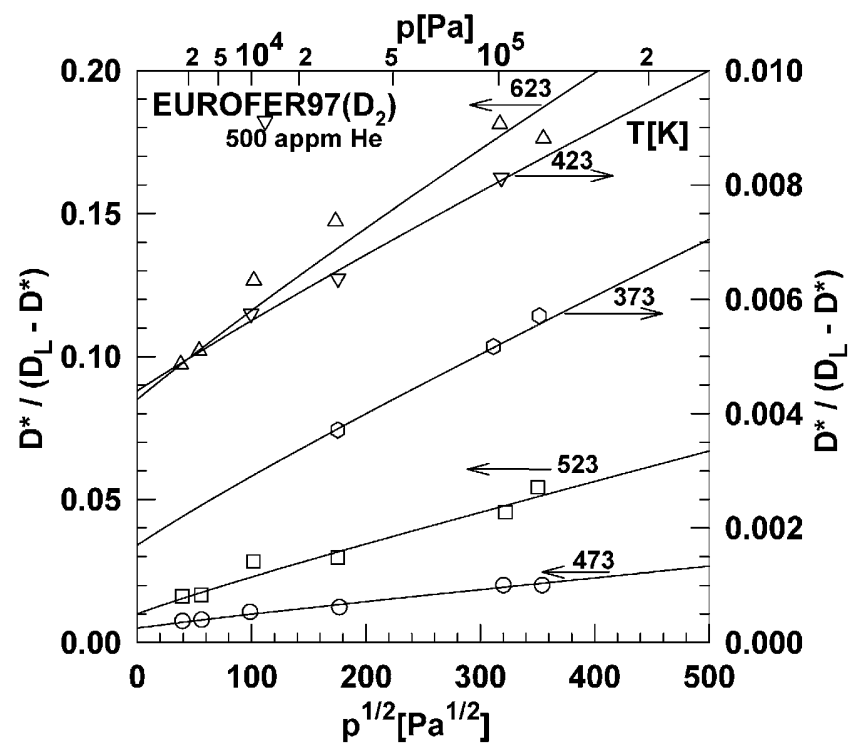

Fig. 4. Pressure dependence of diffusivity $D^{*}$ normalized to lattice diffusivity $D_{L}$ of deuterium in He-implanted EUROFER97 at various temperatures. The slightly curved lines give fits by Eq. (6a). Plotting and evaluation are explained in the text.

The pressure dependence of diffusion coefficients $D^{*}$ of deuterium, normalized to free diffusion in the lattice $D_{L}$, is plotted in Fig. 4. The reason for this type of plotting is explained in Sec. IV. $D_{L}$ is taken from expression (2a). This expression is close to results from Ref. 6, which extended to even higher temperatures.

\section{III.D. Diffusion of Implanted Hydrogen}

When protons are implanted into a foil of thickness $d$ to a depth $r$, which depends on particle energy, the permeating flux to the back surface gradually increases during implantation and eventually reaches a stationary value. Diffusion coefficients of the implanted protons $D^{I}$ can be derived from the half-time $t_{1 / 2}$ of the transient after switching the beam on or off. According to numerical calculations, ${ }^{7} D^{I}$ can be approximated within $\sim 10 \%$ by

$$
D^{I}=\frac{d^{2} \cdot \sin ^{2} \pi \cdot(1-r / d)}{\pi^{2} \cdot \varepsilon \cdot t_{1 / 2}} \quad \text { for } r / d<\frac{1}{2}
$$

and

$$
D^{I}=\frac{(d-r)^{2}}{\varepsilon \cdot t_{1 / 2}} \quad \text { for } r / d>\frac{2}{3} .
$$

The factor $1 / \varepsilon$ takes into account permanent trapping; i.e., it approximates the total released fraction of implanted atoms $(1 / \varepsilon \approx f \cdot d / r$, with $f$ the ratio of permeating to implanted proton flux). $D^{I}$ values taken during beam-on and beam-off periods show small and unsystematic differences. Variations of $D^{I}$ during repeated experiments, which might occur by defect accumulation, are insignificant, because of the small accumulated doses. Data for different material conditions are shown in Fig. 5 for implantation to one-quarter (open), one-half (crossed), and three-quarters (filled) of specimen thickness $d$. The results for unirradiated and for preirradiated material at the highest temperature $\left(350^{\circ} \mathrm{C}\right)$ fall significantly below the respective results from gas diffusion, which are indicated by the lines, confirming reduction of diffusivity by defect production. For all other material conditions and temperatures, differences range within the scatter band of data. The He-implanted-plus-annealed specimens show at the very beginning of $\mathrm{H}$ implantation rather high diffusion coefficients (these are given in Fig. 5), which in the case of deep $\mathrm{H}$ implantation even exceed the values from gas permeation but which decrease by up to an order of magnitude at rather small doses.

\section{III.E. Solubility}

Hydrogen solubility $K^{*}$ as derived from the quotient $P^{*} / D^{*}$ is shown in Fig. 6 as a function of reciprocal temperature. Characteristic values from different pressure ranges are plotted separately, showing only minor pressure dependence. Asymptotic effective molar solubility $K^{*}$ of $\mathrm{D}_{2}$ in EUROFER 97 at temperatures $>250^{\circ} \mathrm{C}$

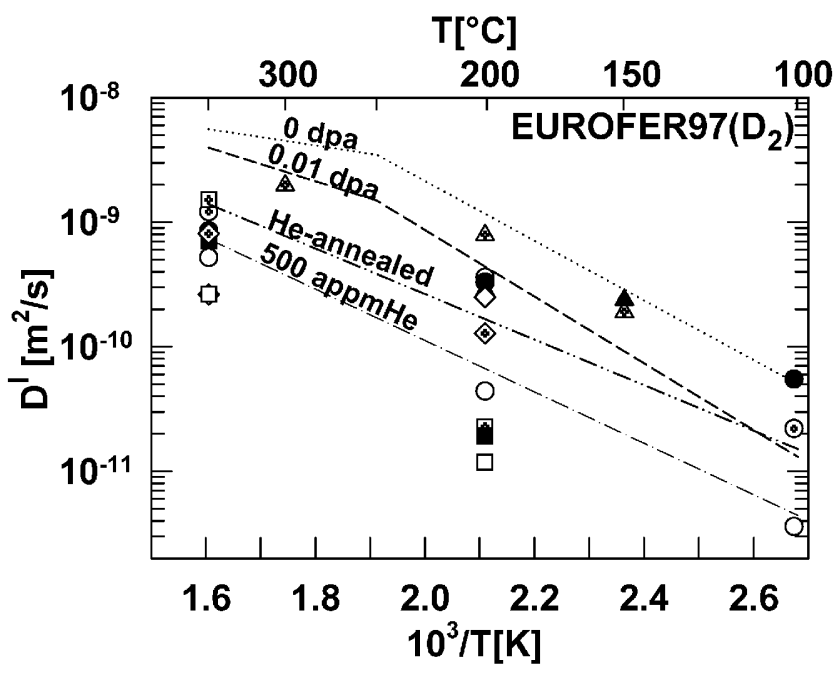

Fig. 5. Diffusion coefficients $D^{I}$ of implanted hydrogen in EUROFER97 (symbols) as a function of reciprocal temperature compared to gas diffusion (lines), for standard condition $(\triangle, \cdots)$, preirradiated to $0.01 \mathrm{dpa}(\bigcirc,--)$, implanted with $500 \mathrm{appm} \mathrm{He}(\square,-\cdot-)$, and $500 \mathrm{appm}$ He implanted plus annealed for $10 \mathrm{~h}$ at $750^{\circ} \mathrm{C}(\diamond$, -..-). Implantation depths were one-quarter (empty), one-half (crosses), and three-quarters (filled) thickness, respectively. 


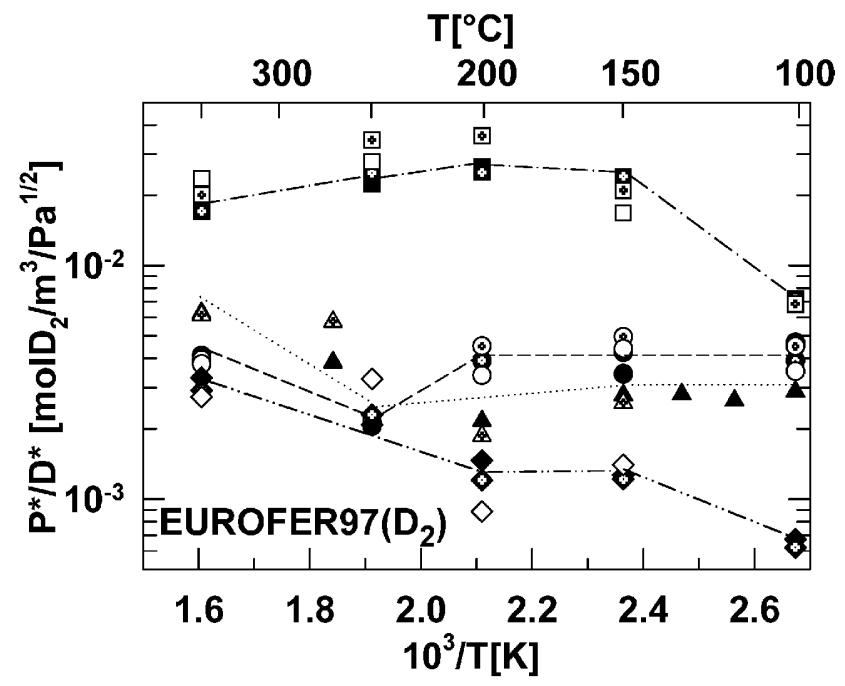

Fig. 6. Temperature dependence of solubility $K^{*}=P^{*} / D^{*}$ of deuterium at pressures from $10^{3}$ to $10^{4} \mathrm{~Pa}$ (open symbols), $3 \times 10^{4} \mathrm{~Pa}$ (open crossed), $10^{5} \mathrm{~Pa}$ (filled crossed), and $1.2 \times 10^{5}$ to $1.5 \times 10^{5} \mathrm{~Pa}$ (filled) in $750-\mu \mathrm{m}$-thick EUROFER97 of standard condition $(\triangle, \cdots)$; in 380 $\mu \mathrm{m}$-thick specimens preirradiated to $0.01 \mathrm{dpa}(\bigcirc,---)$; in 500- $\mu \mathrm{m}$-thick specimens preimplanted to $500 \mathrm{appm}$ $\mathrm{He}(\square,---)$, and in He-implanted specimens annealed for $10 \mathrm{~h}$ at $750^{\circ} \mathrm{C}(\diamond,-\cdots-)$. Lines are included to guide the eye.

and pressures of $\sim 10^{5} \mathrm{~Pa}$ can be described according to Eqs. (1) and (2) by

$$
\begin{aligned}
K_{(\text {std. cond })}^{*} & =2.1 \times \exp (-30800 / R T), \\
K_{(0.01 \mathrm{dpa})}^{*} & =1.1 \times 10^{-1} \exp (-17100 / R T), \\
K_{(500 \text { appm He })}^{*} & =1.0 \times 10^{-1} \exp (-7100 / R T),
\end{aligned}
$$

and

$$
K_{(\text {He } \text { annealed })}^{*}=2.7 \times 10^{-2} \exp (-11200 / R T) .
$$

The lines in Fig. 6 are connecting the data points and do not agree in detail with the Arrhenius behavior described by Eqs. (4a) through (4d), which are derived from Eqs. (1) and (2). For low-defect concentrations it is assumed that the solubility of deuterium in the lattice is not affected by defect production. Therefore, $K_{L}=2 \times$ $K_{(\text {std. cond })}^{*}$ as given by Eq. (4a) is used for atomic lattice solubility throughout.

\section{MODELING}

The dependence of diffusivity on temperature and pressure can be fitted by saturable-trap models, which describe the evolution of the concentrations of free $\left(c_{1}\right)$ and trapped $\left(c_{2}\right)$ hydrogen:

$$
\frac{\partial c_{1}}{\partial t}=D_{L} \cdot \frac{\partial^{2} c_{1}}{\partial x^{2}}-k \cdot c_{1} \cdot\left(c_{T}-c_{2}\right)+q \cdot c_{2}
$$

and

$$
\frac{\partial c_{2}}{\partial t}=k \cdot c_{1} \cdot\left(c_{T}-c_{2}\right)-q \cdot c_{2}
$$

The hydrogen $\left(c_{1}, c_{2}\right)$ and trap concentrations $\left(c_{T}\right)$ describe the respective densities, normalized to the density of lattice atoms $n_{0}\left(\approx 8.3 \times 10^{28} / \mathrm{m}^{3}\right) ; k=4 \pi \cdot r_{T} \cdot D_{L} \cdot n_{0}$ is the trapping rate by a trap on a substitutional lattice site with effective radius $r_{T}$, and $q=\nu / \exp \left(\left(E_{b}+E_{m}\right) / R T\right)$ is the dissociation rate from traps, where $E_{b}$ and $E_{m}$ are the binding energies of hydrogen to a trap and the migration energy in the lattice, respectively, $\nu$ is the attempt frequency, $R$ is the gas constant, and $T$ is the absolute temperature. The lattice diffusivity can be described by $D_{L}=\lambda^{2} \cdot \nu / 6 \cdot \exp \left(E_{m} / R T\right)$. For the nearest neighbor jumps of hydrogen on tetrahedral interstitial sites in a bodycentered-cubic (bcc) lattice, the lattice constant $a=$ $\left(2 / n_{0}\right)^{1 / 3}$, the jump distance $\lambda$ is $a / \sqrt{8}=1 / 2^{7 / 6} n_{0}^{1 / 3}$, and $r_{T}$ for the 24 nearest interstitial sites is $a \sqrt{5} / 4=$ $\sqrt{5} / 2^{5 / 3} n_{0}^{1 / 3}$. It was shown in Ref. 8 that the effective diffusion coefficient $D^{*}$ is related to the asymptotic lattice diffusivity $D_{L}$ by

$$
\begin{aligned}
D^{*}=D_{L} /(1 & +\frac{3 \cdot \alpha}{\beta} \\
\times & {\left.\left[\left(1+\frac{2}{\beta} \cdot\left(1-\left(1+\frac{1}{\beta}\right) \cdot \ln (1+\beta)\right)\right)\right]\right) }
\end{aligned}
$$

with $\alpha=\left(c_{T} / c_{L}\right) \exp \left(E_{b} / R T\right)$ and $\beta=\left(c_{H} / c_{L}\right) \exp \left(E_{b} /\right.$ $R T)$, where $c_{L}$ is the number density of sites for diffusing hydrogen atoms normalized to $n_{0}$, and equal to 6 for diffusion on tetrahedral sites in a bcc lattice, and $c_{H}$ is the number density of hydrogen atoms on the entrance side, normalized to $n_{0}$, i.e., $c_{H}=K_{L} \cdot p^{1 / 2} \cdot \mathrm{Av} / n_{0}$ (Sievert's law, $\mathrm{Av}=$ Avogadro's number), with $K_{L}$ taken from expression (4a). This leaves $c_{T}$ and $E_{b}$ as parameters for fitting expression (6a) to the data, indicated by the lines in Fig. 4. It can be shown that the square brackets in Eq. (6a) can be approximated within $15 \%$ by $\beta /(3+\beta)$, i.e.,

$$
D^{*} \approx D_{L} /\left(1+\frac{3 \cdot \alpha}{3+\beta}\right)
$$

or

$$
\frac{D^{*}}{D_{L}-D^{*}} \approx \frac{1}{\alpha}+\frac{\beta}{3 \alpha}
$$

This means that an almost linear plot is expected for $D^{*} /\left(D_{L}-D^{*}\right)$ versus $c_{H}$, i.e., versus the square root of pressure, as shown in Fig. 4. The asymptotic behavior at high pressures when traps approach saturation $(\beta \gg 1)$ 
for expression (6a) as well as for the approximation (6b) is given by

$$
\begin{aligned}
D^{*} /\left(D_{L}-D^{*}\right) & =\beta / 3 \cdot \alpha=c_{H} / 3 \cdot c_{T} \\
& =\left(K_{L} \cdot p^{1 / 2} \cdot \mathrm{Av}\right) / 3 \cdot c_{T} \cdot n_{0} ;
\end{aligned}
$$

i.e., the slope in Fig. 4 yields $K_{L} \cdot \mathrm{Av} / 3 \cdot c_{T} \cdot n_{0}$, from which $c_{T}$ can be directly approximated. On the other hand, for the behavior at low pressures $(\beta \ll 1)$, i.e., for low trap occupancy, the extrapolated ordinate value in Fig. 4 from Eq. (6a) as well as from Eq. (6b) is given by ${ }^{\mathrm{a}}$

$$
D^{*} /\left(D_{L}-D^{*}\right)=1 / \alpha=\left(c_{L} / c_{T}\right) \exp \left(-E_{b} / R T\right),
$$

yielding $E_{b}$ when $c_{T}$ from the slope [expression (7a)] is inserted.

The parameters $c_{T}$ and $E_{b}$, derived from Eqs. (7a) and $(7 b)$, are plotted as a function of reciprocal temperature in Fig. 7. These values, derived for individual data points, partially differ from values obtained from the overall linear fits, included, for example, in Fig. 4, while the product $c_{T} \exp \left(E_{b} / R T\right)$ from both evaluations is virtually the same. These differences indicate the uncertainty of this fitting. Nevertheless, the qualitative trend in both sets of parameters is the same: Trap concentration $c_{T}$ is lowest in the standard condition, increases after 0.01 dpa irradiation, and increases even more after He implantation, while it is reduced by annealing of the implanted specimens. For all conditions, $c_{T}$ is decreasing between 150 and $200^{\circ} \mathrm{C}$ by about one order of magnitude. Binding energy $E_{b}$ clearly increases with temperatures below 50 $\mathrm{kJ} / \mathrm{mol}$ at $100^{\circ} \mathrm{C}$ to above $70 \mathrm{~kJ} / \mathrm{mol}$ at $350^{\circ} \mathrm{C}$ for all conditions, with the exception of the $0.01 \mathrm{dpa}$ specimen, which shows some leveling off above $200^{\circ} \mathrm{C}$.

The plotting in Fig. 4 has advantages compared to the commonly used plotting of $D_{L} /\left(D^{*}-1\right)$ versus $p^{-1 / 2}$, e.g., Refs. 2 and 9, which only have simple dependencies at asymptotic pressures, namely, a constant value at very low pressures and a linear decrease at very high pressures. As both pressure conditions usually are not met in experiments, fitting of this relation is subject to significant uncertainties.

\section{DISCUSSION}

The trap concentrations $c_{T}$ and binding energies $E_{b}$ in standard condition EUROFER97 and after preirradiation reasonably agree with values derived for $\mathrm{F} 82 \mathrm{H}-\mathrm{mod}$

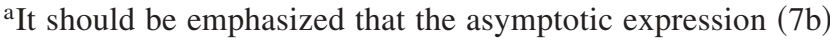
applies only to low pressures, when trap occupancy is far from saturation. As can be seen from Fig. 4, this situation is reached at low temperatures only for decreasingly low pressures, e.g., at $10^{3} \mathrm{~Pa}$ for $150^{\circ} \mathrm{C}$. On the other hand at higher temperatures, when expression (7a) may be applicable at pressures of $\sim 10^{5} \mathrm{~Pa}, D^{*}$ approaches $D_{L}$, causing increasing errors of the evaluation.
}

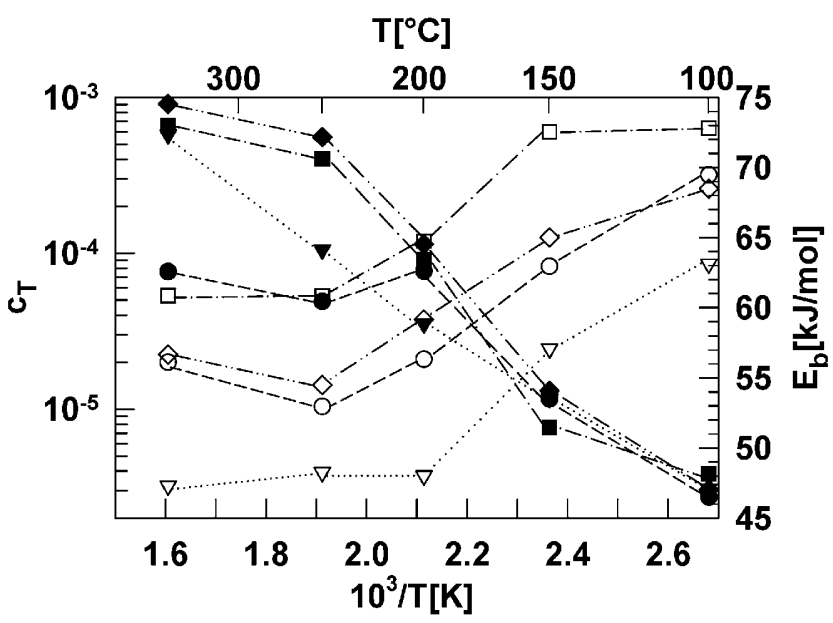

Fig. 7. Fit parameters $c_{T}$ (open symbols, left-hand ordinate) and $E_{b}$ (filled, right-hand ordinate) for $\mathrm{D}_{2}$ diffusivities, derived from expressions (7a) and (7b), in 500- $\mu \mathrm{m}-$ thick EUROFER97 specimens of standard condition $(\nabla, \cdots)$, in $380-\mu m$-thick specimens preirradiated to $0.01 \mathrm{dpa}(\mathrm{O},---)$, in $500-\mu \mathrm{m}$-thick specimens preimplanted to $500 \mathrm{appm} \mathrm{He}(\square,---)$, and in He-implanted specimens annealed for $10 \mathrm{~h}$ at $750^{\circ} \mathrm{C}(\diamond,-\cdots-)$. Lines are included to guide the eye.

(Ref. 3). For both materials $E_{b}$ are of similar magnitude and increase with temperature, indicating that the behavior of hydrogen in steels, also in the standard condition, cannot be sufficiently described by only one type of trap. On the other hand, evaluation becomes increasingly uncertain with increasing numbers of parameters. The $c_{T}$ values of He-implanted specimens on the average exceed those of the preirradiated specimens by a factor of $\sim 3$. This is slightly less than the factor of 6 ratio of displacement doses: 0.065 versus $0.01 \mathrm{dpa}$. This shows that trap formation is dominated by displacement defects, while the implanted helium may have only a secondary effect. The clear increase of $c_{T}$ by irradiation and He implantation is opposed by minor changes of $E_{b}$. This observation, that defect production mainly enhances trap concentration but has much less effect on effective binding energy, has also been reported for deformed iron. ${ }^{10}$ The increase of $E_{b}$ and decrease of $c_{T}$ by annealing of He-implanted specimens indicates partial recovery and formation of stronger traps by clustering of defects.

Characteristic parameters of permeability, $P^{*}=$ $P_{0} \exp \left(-Q_{p} / R T\right)$, and diffusivity, $D_{L}=D_{L 0} \exp \left(-\Delta H_{L} /\right.$ $R T$ ), for EUROFER97, F82H-mod (Ref. 3), and MANET-II (Ref. 2) are listed in Table I. Trapping is characterized by the ratios of diffusivity $D^{*}$ at 150 and $300^{\circ} \mathrm{C}$. Most of the data show a trend with $\mathrm{Cr}$ content, e.g., the increase of $Q_{p}$ with $c_{C r}$ is in agreement with a relation derived previously. ${ }^{5}$ With respect to diffusivity, both $D_{L 0}$ and $\Delta H_{L}$ decrease with increasing chromium content. Also, increasing trapping with $c_{\mathrm{Cr}}$ is clearly 
TABLE I

Characteristic Parameters of Permeability and Diffusivity of High Chromium Steels in the European Fusion Program

\begin{tabular}{|c|c|c|c|c|c|c|}
\hline Material & $\underset{(\mathrm{wt} \%)}{\mathrm{Cr}}$ & $\begin{array}{c}P_{0} \\
{\left[\mathrm{~mol} \mathrm{D}_{2} /\left(\mathrm{m} \cdot \mathrm{s} \cdot \mathrm{Pa}^{1 / 2}\right)\right]}\end{array}$ & $\begin{array}{c}Q_{p} \\
(\mathrm{~kJ} / \mathrm{mol})\end{array}$ & $\begin{array}{c}D_{L 0} \\
\left(\mathrm{~m}^{2} / \mathrm{s}\right)\end{array}$ & $\begin{array}{c}\Delta H_{L} \\
(\mathrm{~kJ} / \mathrm{mol})\end{array}$ & $D^{*}(300) / D^{*}(150)$ \\
\hline F82H-mod & 7.7 & $5.7 \times 10^{-8}$ & 41.3 & $1.8 \times 10^{-7}$ & 14.1 & 7.5 \\
\hline EUROFER97 & 9.0 & $1.4 \times 10^{-7}$ & 42.5 & $6.6 \times 10^{-8}$ & 12.8 & 25 \\
\hline MANET-II & 10.3 & $7.0 \times 10^{-8}$ & 43.4 & $4.5 \times 10^{-8}$ & 10.0 & 50 \\
\hline
\end{tabular}

indicated by the ratios in the last column. This increased deviation of diffusivities at low temperatures from the values of pure iron is clearly seen in Fig. 8.

\section{SUMMARY}

A summary of the results of this study is as follows:

1. Permeability of deuterium in EUROFER97 is slightly reduced by preirradiation, more strongly by implanted He, and even more after formation of He bubbles.

2. Diffusivity is slightly reduced by preirradiation and more strongly by helium implantation, but the effect of helium is reduced by clustering.

3. Solubility as derived from the ratio of permeability, and diffusivity shows only a minor effect of preirra-

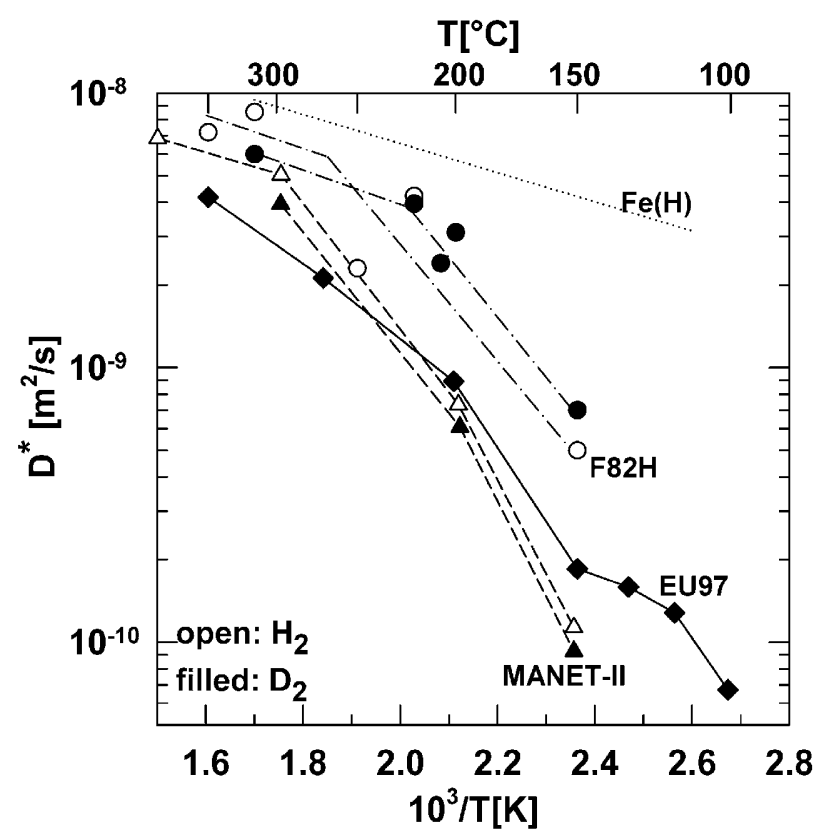

Fig. 8. Diffusion coefficients of deuterium in $750-\mu$ m-thick EUROFER97, compared to $\mathrm{H}$ in pure $\mathrm{Fe}$, and compared to $\mathrm{H}$ and D in 817- $\mu$ m-thick F82H-mod (Ref. 3) and in $810-\mu \mathrm{m}$-thick MANET-II (Ref. 2), respectively. diation. It is enhanced by implanted helium, but this enhancement disappears upon clustering.

4. Diffusion coefficients derived from implanted hydrogen for all material conditions are generally within the scatter band of data from gas diffusion or are slightly lower.

5. Trap concentrations, as tentatively derived from a saturable-trap model, increase by preirradiation and decrease with increasing temperature.

6. Effective trap binding energies are only slightly affected by irradiation or implantation but increase with temperature, indicating a complex trap structure.

7. Permeation is not affected by simultaneous irradiation if temperature changes induced by irradiation are taken into account.

\section{REFERENCES}

1. P. JUNG, Fusion Technol., 33, 63 (1998).

2. F. WEDIG and P. JUNG, J. Nucl. Mater., 245, 138 (1997).

3. F. SCHLIEFER, C. LIU, and P. JUNG, J. Nucl. Mater., 283-287, 540 (2000).

4. J. P. BIERSACK and L. G. HAGGMARK, Nucl. Instrum. Methods, 174, 93 (1980); see also J. F. ZIEGLER, "Manual of TRIM Version 95.4” (Mar. 1995) (unpublished).

5. P. JUNG, J. Nucl. Mater., 238, 189 (1996).

6. A. AiEllo, G. BENAMATI, L. BERTINI, and M. BEGHINI, SM-A-R-001, ENEA, July 31, 2003.

7. P. JUNG, Nucl. Instrum. Methods Phys. Res. B, 91, 362 (1994).

8. A. McNABB and P. K. FOSTER, Trans. Met. Soc. AIME, 227, 619 (1963).

9. H. H. JOHNSON, Metal. Trans. A, 19, 2371 (1988).

10. H. H. JOHNSON and R. W. LIN, in Hydrogen Effects in Metals, p. 3, I. M. BERNSTEIN and A. W. THOMPSON, Eds., Met. Soc. AIME (1981). 